This item was submitted to Loughborough's Research Repository by the author.

Items in Figshare are protected by copyright, with all rights reserved, unless otherwise indicated.

\title{
Evaluating the safety impact of connected and autonomous vehicles on motorways
}

\section{PLEASE CITE THE PUBLISHED VERSION}

https://doi.org/10.1016/j.aap.2018.12.019

\section{PUBLISHER}

(c) Elsevier

VERSION

AM (Accepted Manuscript)

\section{PUBLISHER STATEMENT}

This paper was accepted for publication in the journal Accident Analysis and Prevention and the definitive published version is available at https://doi.org/10.1016/j.aap.2018.12.019.

LICENCE

CC BY-NC-ND 4.0

\section{REPOSITORY RECORD}

Papadoulis, Alkis, Mohammed Quddus, and Marianna Imprialou. 2019. "Evaluating the Safety Impact of Connected and Autonomous Vehicles on Motorways". figshare. https://hdl.handle.net/2134/36819. 


\section{Evaluating the Safety Impact of Connected and Autonomous \\ Vehicles on Motorways}

4 Alkis Papadoulis MEng*

5 PhD Candidate

6 School of Architecture, Building \& Civil Engineering

7 Loughborough University

8 Loughborough LE11 3TU

9 United Kingdom

10 Tel: $+44(0) 1509223422$

11 Email: L.A.Papadoulis@,lboro.ac.uk

12 Professor Mohammed Quddus

13 Professor of Intelligent Transport Systems

14 School of Architecture, Building \& Civil Engineering

15 Loughborough University

16 Loughborough LE11 3TU

17 United Kingdom

18 Tel: +44 (0) 1509228545

19 Email: M.A.Quddus@,1boro.ac.uk

20

21 Dr Marianna Imprialou

22 Lecturer (Assistant Professor)

23 School of Architecture, Building \& Civil Engineering

24 Loughborough University

25 Loughborough LE11 3TU

26 United Kingdom

27 Tel: +44 (0) 1509223422

28 Email: M.Imprialou@lboro.ac.uk

29

$30 *$ Corresponding author 
Papadoulis, Quddus, Imprialou

\section{$1 \quad$ ABSTRACT}

2 Recent technological advancements bring the Connected and Autonomous Vehicles (CAVs)

3 era closer to reality. CAVs have the potential to vastly improve road safety by taking the human

4 driver out of the driving task. However, the evaluation of their safety impacts has been a major

5 challenge due to the lack of real-world CAV exposure data. Studies that attempt to simulate

6 CAVs by using either a single or integrating multiple simulation platforms have limitations,

7 and in most cases, consider a small element of a network (e.g. a junction) and do not perform

8 safety evaluations due to inherent complexity. This paper addresses this problem by developing

9 a decision-making CAV control algorithm in the simulation software VISSIM, using its

10 External Driver Model Application Programming Interface. More specifically, the developed

11 CAV control algorithm allows a CAV, for the first time, to have longitudinal control, search

12 adjacent vehicles, identify nearby CAVs and make lateral decisions based on a ruleset

13 associated with motorway traffic operations. A motorway corridor within M1 in England is

14 designed in VISSIM and employed to implement the CAV control algorithm. Five simulation

15 models are created, one for each weekday. The baseline models (i.e. CAV market penetration:

$160 \%$ ) are calibrated and validated using real-world minute-level inductive loop detector data and

17 also data collected from a radar-equipped vehicle. The safety evaluation of the proposed

18 algorithm is conducted using the Surrogate Safety Assessment Model (SSAM). The results

19 show that CAVs bring about compelling benefit to road safety as traffic conflicts significantly

20 reduce even at relatively low market penetration rates. Specifically, estimated traffic conflicts

21 were reduced by $12-47 \%, 50-80 \%, 82-92 \%$ and $90-94 \%$ for $25 \%, 50 \%, 75 \%$ and $100 \%$ CAV

22 penetration rates respectively. Finally, the results indicate that the presence of CAVs ensured

23 efficient traffic flow.

24 Keywords: Connected and Autonomous Vehicles; Traffic Microsimulation; Road Safety;

25 Surrogate Safety Assessment Model; VISSIM; External Driver Model; 
Papadoulis, Quddus, Imprialou

\section{INTRODUCTION}

Connected and Autonomous vehicles (CAVs) will be a reality in the near future and have the potential to reduce traffic congestion, road crashes and vehicle emissions (Poczter and Jankovic, 2014; Fagnant and Kockelman, 2015). The concept of CAVs has generated high expectations regarding safety performance of future road transport systems, as $94 \%$ of crashes involve human error as a contributing factor (Singh, 2015). Based on this figure, few previous studies have evaluated the potential safety impact of CAVs, estimating a safety benefit of approximately $90 \%$ during the fully autonomous era. However, this level of impact has not been confirmed yet due to lack of data.

For this reason, the vast majority of automotive manufacturers have ongoing projects on CAVs and field operating trials are being carried out in both motorways and urban environments (e.g. Waymo project (Waymo, 2016)). These trials have proven that CAVs will introduce a spectrum of unprecedented challenges and will bring a multifaceted transformation to the existing road network. For instance, whether existing motorway and urban infrastructure can accommodate CAVs is yet to be fully elucidated. Additionally, the communication standards and a unified protocol between existing infrastructure and CAVs have not been clarified yet. Furthermore, the inherent challenges arising from the interaction between human driven and CAVs during the transition period are largely unknown (Reich, 2013). Moreover, the compatibility between software of different CAV manufacturers is uncertain and this might affect their operations and co-operative decision making at a corridor or a network level (Anderson et al., 2014).

Despite the real-world CAV trials and the advancements in knowledge, CAV fleet data are not easily obtainable. This is one of the primary reasons why existing part of literature has mainly speculated the impacts of CAVs (Anderson et al., 2014; Fagnant and Kockelman, 2015; Kim et al., 2015). More recently, research has focused on CAV simulation which may be perhaps the only viable alternative method to evaluate the impact of CAVs and can provide valuable initial insight into the implementation of CAVs. However, recent attempts to simulate CAVs found in the literature have limitations.

This study advances the understanding of the impacts of CAVs by conducting a comprehensive CAV safety evaluation using traffic microsimulation. For this purpose, a decision-making CAV control algorithm is developed in the External Driver Model Application Programming Interface (API) of PTV VISSIM traffic micro-simulation software. The proposed algorithm is assigned to a specific vehicle category in the simulation software and is tested in a calibrated motorway model. The corridor-level safety impact of CAVs is finally evaluated using the Surrogate Safety Assessment Model (SSAM).

The paper is organised as follows: In the next section, existing literature on CAV simulation and its differences from this study are presented. A detailed description of the road segment drawn in VISSIM, the functionality of the CAV control algorithm and the calibration and validation process follow next. The fourth section of the paper presents the results obtained from the simulation, discusses about their contiguity to existing literature, presents this study's limitations and proposes future directions for CAV simulation research. Finally, the last section summarises the conclusions of the research.

\section{PREVIOUS RESEARCH}

The purpose of this literature review is to critically analyse methods that have been applied in studies to derive the range and scale of the impact of CAVs with a special focus on safety. It is 
Papadoulis, Quddus, Imprialou

1 worthwhile to point out that not all the studies reviewed evaluate the safety impact of CAVs.

2 Nonetheless, the method and data employed in each of the studies are analysed. It must be

3 emphasized that, for consistency, studies mentioning or implying both vehicle automation and

4 connectivity are considered in this section.

5 The two major approaches identified in the literature to estimate CAV impacts are, so far, based

6 either based on historical or simulated data.

\subsection{Studies employing historical data}

8 Recent studies have attempted to predict the potential safety impact of CAVs using historical 9 crash data (Hayes, 2011; Silberg et al., 2012; Fagnant and Kockelman, 2015). Based on the 10 number of crashes that include human error as a contributing factor and under the assumption 11 that this factor will not exist in autonomous road environments, it is suggested that a $90 \%$ 12 reduction in crash rates can be achieved with the introduction of CAVs (Fagnant and 13 Kockelman, 2015). Alternatively, by comparing CAV implementation to the implementation 14 of automated technologies in aviation or rail, studies conclude that road crash rates could be as low as those of aviation and rail, ultimately reaching $1 \%$ of the current figures (Hayes, 2011). Although these approaches have provided a useful starting point in understanding the scale of safety benefits, they are based on a series of assumptions that may limit their capability in producing reliable outcomes.

\subsection{Studies applying CAV simulation}

Research has focused on CAV simulation during the past few years. Based on the approach of CAV simulation, the studies can be categorized into two groups. The first approach includes studies that use custom-built simulation frameworks (Figueiredo et al., 2009; Noort, Arem and Park, 2010; O'Hara et al., 2012; Pereira and Rossetti, 2012). These studies aim to achieve detailed CAV simulation by creating an integrated multi-level simulation platform, which in most cases include traffic, sensor (robotics) and network simulators. Simulation software used in these studies includes VISSIM, SUMO and Mas T2erLab for traffic simulation, USARSIM, MDDVS PreScan for robotics simulation and NS2 or NS3 for network simulation. The complexity and the unique design of these proposed simulation frameworks makes the results difficult to interpret, less reliable and the comparison challenging (Li et al., 2013). Additionally, although these studies could simulate CAVs accurately, they had high computational needs, a fact that limited the scale of the conducted experiments significantly, compared to simpler simulation applications.

The alternative approach includes studies that use a traffic micro-simulation software and its extensions (e.g. API or COM interface). This method usually introduces simplifications, such as an indirect simulation of vehicle sensors or vehicle communication. This kind of simplifications, however, has a small effect on the calculation of the network- or corridor-level impact of CAVs as the functionality of sensors can be indirectly programmed sufficiently. Most of the studies adopting this method manage to develop large scale experiments and produced significant results. This category of studies comprises of differences in the definition of CAVs' operational characteristics and therefore their outcomes are not necessarily comparable.

41 Roncoli et al. (2015) used AIMSUN to model vehicle automation and vehicle to infrastructure 42 (V2I) communication in a motorway with ramp metering and variable speed limit setting. Their 43 study relied on a Traffic Management Centre (TMC) for lane changing decisions. However, 44 this is only a partial representation of CAV control behaviour, as although CAVs might be able 
Papadoulis, Quddus, Imprialou

1 to follow suggestions from a centralised TMC in the future, the major challenge relates to their

2 ability to sense their surroundings and make independent decisions accordingly.

3 Furthermore, Park et al. (2012) used PARAMICS to evaluate the impact of vehicle automation 4 on ramp management through an algorithm that proposed advisory lane changes upstream of 5 ramp merging areas of the motorway. The study could suggest advisory lane changes on ramp 6 areas and could therefore reduce only merging-related conflicts.

7 Finally, there is a series of studies that used VISSIM to simulate CAVs. Li et al. (2013) used 8 the External Driver Model API of VISSIM to model autonomous intersection control. They 9 transferred the VISSIM generated vehicle trajectories data into the SSAM software to estimate 10 the number of generated conflicts. The autonomous intersection control proved to be highly 11 effective producing only one traffic conflict in 1,800 seconds of simulation. However, this 12 method is designed specifically for intersections and is not transferrable to a network or 13 corridor level.

14 ATKINS (2016) used VISSIM's COM interface in order to evaluate the impact of CAVs in urban and motorway networks with respect to the efficiency of traffic flow. Despite using a wide range of CAV market penetration rates, time headways between vehicles and CAVorientated parameters, CAV decisions were ultimately made through the default driver model of VISSIM. Similarly, Stanek et al. (2018) changed the default Wiedemann 99 driving model parameters in VISSIM in order to simulate connected and autonomous driving behaviour according to values found in the literature. The alteration of the default driver model parameters of VISSIM (e.g. CC1; safety distance to the preceding vehicle) however, cannot be directly connected to or imply full vehicle automation and connectivity. Also, a possible use of this method for safety evaluation could show inaccurately increased simulated traffic conflicts due to the stricter headway safety parameters of VISSIM. Jeong et al (2017) also used VISSIM's $\mathrm{COM}$ interface. They proposed a CAV algorithm that controlled the longitudinal movement of CAVs during the simulation based on surrounding traffic conditions. Their proposed algorithm showed encouraging network and individual-level safety results and provided useful insights. However, it inevitably included assumptions regarding the longitudinal control of the CAV and did not include a lateral control algorithm.

\subsection{Use of Traffic Simulation for Safety Evaluation}

The use of traffic micro-simulation for safety evaluation has been questioned about its ability to reproduce real-world traffic conditions reliably (Tarko, 2005; Saunier et al, 2007). However, studies are supporting the use of microsimulation for safety evaluation purposes (Fan et al., 2013; Huang et al., 2013; Essa and Sayed, 2015; Shahdah, Saccomanno and Persaud, 2015). These studies use traffic micro-simulation for safety evaluation through the SSAM, developed by the Federal Highway Administration (FHWA) and manage to obtain promising results through safety oriented two-stage calibration methods. ; For example, Fan et al., (2013) used traffic conflicts as a measure of safety to calibrate their simulation network. They concluded that conflicts calculated through SSAM are similar to those measured in the field using traditional traffic conflicts techniques. This two-stage calibration method can improve the representativeness of the simulated data; however, it is sensitive to observation error as the characterisation of vehicle conflicts may be subjective. A two-stage calibration is applied in this paper as well and is described in the following section.

44 In summary, it is difficult to reliably predict the safety impact of this disruptive paradigm shift 45 on existing transport systems as CAVs are not yet present in traffic streams. There is a need to 
Papadoulis, Quddus, Imprialou

1 develop a CAV control algorithm that can simulate CAVs containing as few assumptions as 2 possible and implement it in an integrated roadway environment to examine the safety impact. 3 This paper takes a step towards this direction by developing an integrated algorithm in VISSIM, 4 in order to evaluate the corridor-level safety impact of CAVs.

\section{METHOD}

The evaluation of the safety impacts of CAVs in this paper, is conducted on a motorway segment designed in VISSIM. CAVs are represented using an external CAV driver model API written in $\mathrm{C}++$ programming language. The external driver model was assigned to a specific vehicle type in VISSIM. This section describes the design of the motorway model, its calibration and validation, explains the functionality of the proposed CAV control algorithm and the interaction between VISSIM and SSAM. The overall architecture of the proposed simulation framework is presented in Figure 1.

\section{SIMULATION PROCESS}
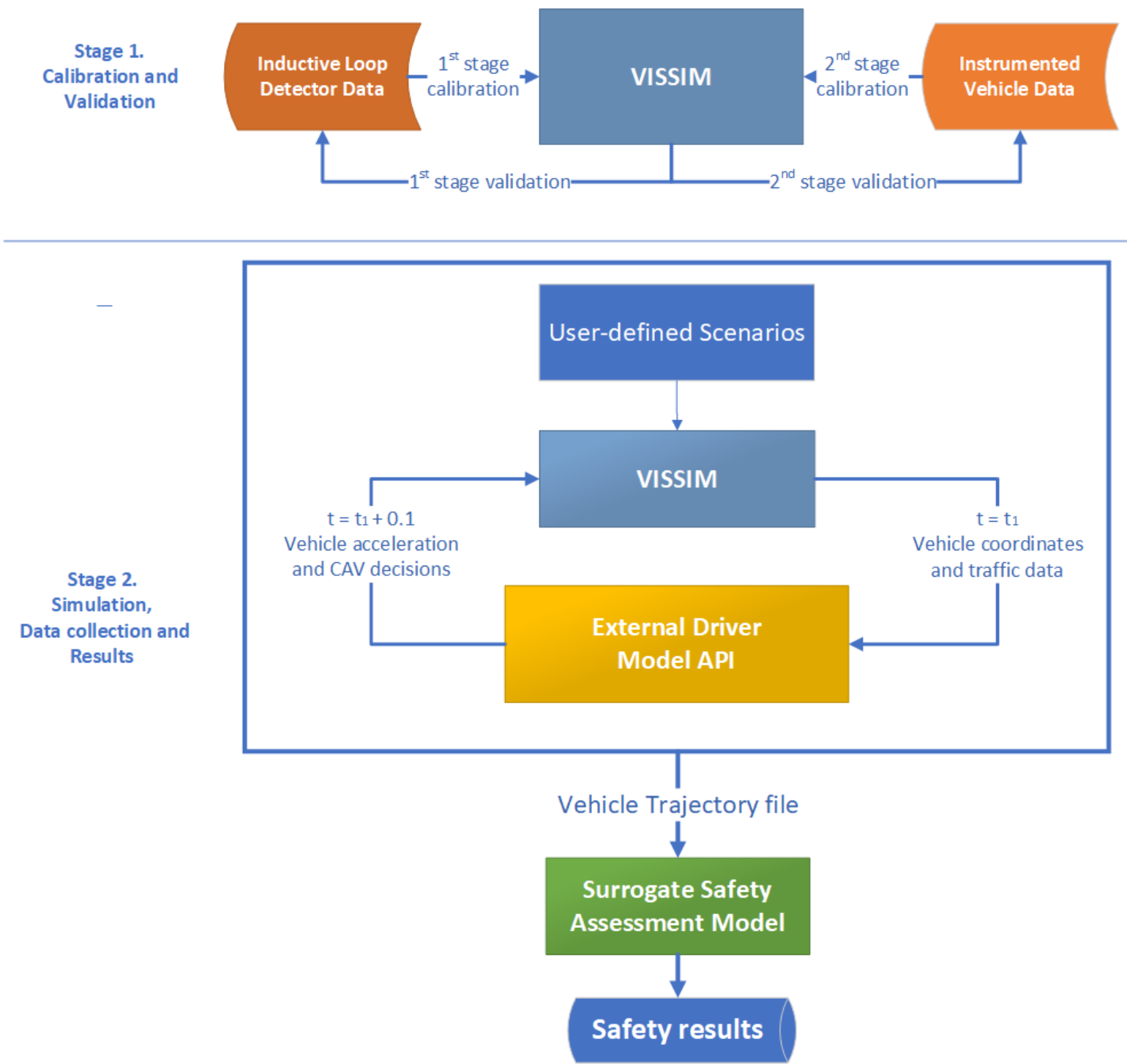

14 Figure 1 Architecture of the CAV microsimulation framework 
Papadoulis, Quddus, Imprialou 4

\subsection{The Microsimulation Platform}

After conducting a critical evaluation of most available traffic micro-simulation packages and taking into account the advantages and disadvantages of all available traffic simulators (Pereira and Rossetti, 2012), the widely-used traffic micro-simulation software package PTV VISSIM 9.0 combined with its External Driver Model Application Programming Interface (API) was chosen for this study. A three-lane motorway section (M1 Junctions 19-21, located between Leicester and Rugby in the United Kingdom) was chosen as a test-bed. Initially, by using an aerial photograph, both directions of the road segment were drawn. Lane width and the length of links, merging and diverging areas were drawn according to the aerial photograph. The merging and diverging areas were designed following guidelines from literature specialising in motorway merge areas (e.g. Fan et al., 2013; Whaley, 2016). The total length of the mainline corridor designed was $44.27 \mathrm{~km}$, contained eight on and off-ramps in total and six vehicle input points (see Figure 2). The model designed, did not contain the roundabout at Junction 20.

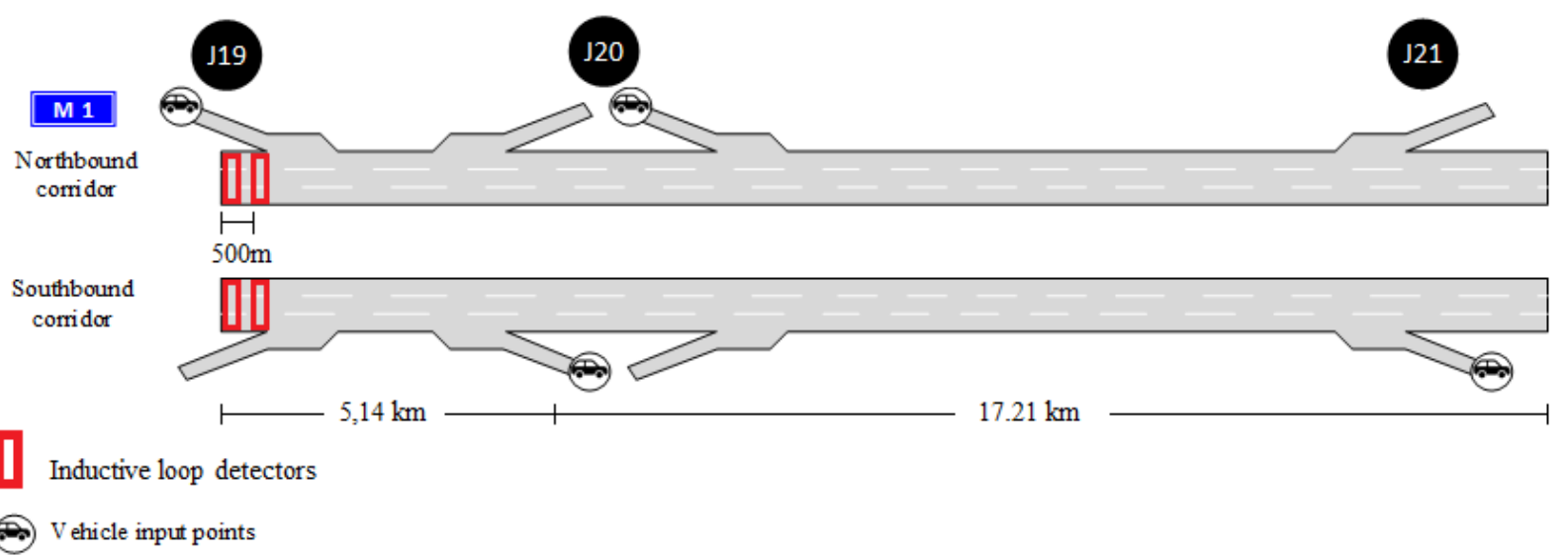

Figure 2 Outline of the simulated motorway segment

\section{Driver Behaviour}

Vehicle movement in VISSIM is determined by other vehicles' movement and surrounding infrastructure. The driver and the vehicle are considered as one entity and can be assumed to be in one of the four driving modes - free driving, approaching, braking and following. The default human driver behaviour is represented using the Wiedemann 99 or Wiedemann 74 psycho-social model (PTV AG, 2015). A calibrated Wiedemann 99 driving model was used to simulate human driving behaviour in this paper, since it was recommended by VISSIM for interurban traffic and contained more adjustable driving behaviour parameters which could contribute to a more accurate safety-orientated calibration of the baseline model. A list of the aforementioned parameters is presented in Table 1.

Table 1 Adjustable driving behaviour parameters in PTV VISSIM 9.0

\begin{tabular}{|l|l|l|}
\hline Parameter & Unit & Description \\
\hline CC0 & $\mathrm{m}$ & $\begin{array}{l}\text { Standstill distance: the average desired standstill distance between two } \\
\text { vehicles. It has no variation. }\end{array}$ \\
\hline CC1 & $\mathrm{s}$ & Time headway: is the time (in seconds) that a driver wants to keep. \\
\hline
\end{tabular}


Papadoulis, Quddus, Imprialou

\begin{tabular}{|c|c|c|}
\hline $\mathrm{CC} 2$ & $\mathrm{~m}$ & $\begin{array}{l}\text { 'Following' variation: restricts the longitudinal oscillation or how much } \\
\text { more distance than the desired safety distance a driver allows before he } \\
\text { intentionally moves closer to the car in front. }\end{array}$ \\
\hline $\mathrm{CC} 3$ & $\mathrm{~s}$ & $\begin{array}{l}\text { Threshold for entering car following mode in VISSIM: controls the start } \\
\text { of the deceleration process, i.e. when a driver recognizes a preceding } \\
\text { slower vehicle. }\end{array}$ \\
\hline $\mathrm{CC} 4$ & $\mathrm{~m} / \mathrm{s}$ & \multirow{2}{*}{$\begin{array}{l}\text { Following' thresholds: control the speed differences during the } \\
\text { 'Following' state. Smaller values result in a more sensitive reaction of } \\
\text { drivers to accelerations or decelerations of the preceding car }\end{array}$} \\
\hline $\mathrm{CC} 5$ & $\mathrm{~m} / \mathrm{s}$ & \\
\hline CC6 & $1 /(\mathrm{m} * \mathrm{~s})$ & $\begin{array}{l}\text { Speed dependency of oscillation: influence of distance on speed oscillation } \\
\text { while in following process. }\end{array}$ \\
\hline $\mathrm{CC} 7$ & $\mathrm{~m} / \mathrm{s}^{2}$ & Oscillation acceleration: actual acceleration during the oscillation process. \\
\hline $\mathrm{CC} 8$ & $\mathrm{~m} / \mathrm{s}^{2}$ & Standstill acceleration: desired acceleration when starting from standstill \\
\hline CC9 & $\mathrm{m} / \mathrm{s}^{2}$ & Acceleration at $80 \mathrm{~km} / \mathrm{h}$ : desired acceleration at $80 \mathrm{~km} / \mathrm{h}$ \\
\hline
\end{tabular}

2 For CAV simulation, the External Driver Model API of VISSIM allows the user to integrate 3 an external application in the simulation. For this study, a user-defined driving behaviour was 4 developed using $\mathrm{C}++$ programming language and a compatible dynamic link library (dll) 5 compiler, Microsoft Visual Studio. The driving behaviour dll, replaced the internal driving 6 behaviour with CAV driving behaviour and was assigned only to the CAV-type vehicles in 7 VISSIM. VISSIM passes the state of a vehicle and its surroundings to the external application 8 and the dll computes the behaviour of the vehicle for the next time step. The flowchart of the 9 proposed CAV control algorithm is shown in Figure 3 and is described in the following 10 paragraphs. 
Papadoulis, Quddus, Imprialou

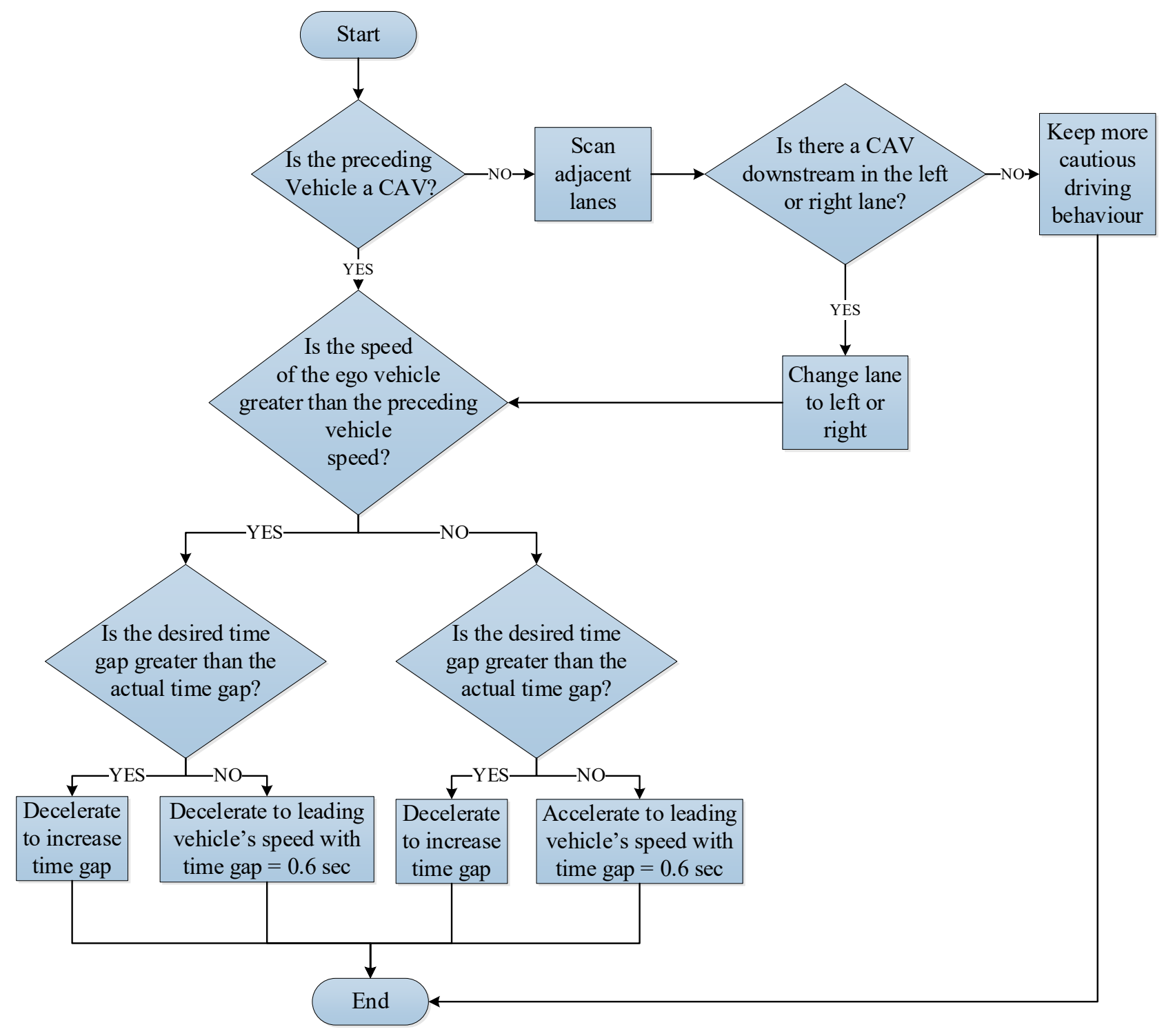

2 Figure 3 Flowchart of the proposed CAV control algorithm.

3

4 Longitudinal Movement

5 This study assumes that CAVs are able to drive closer to their preceding vehicles compared to

6 human drivers, ultimately forming vehicle platoons with other CAVs. The accepted car-

7 following time-gap chosen for this study was 0.6 seconds, which is in-line with relevant

8 literature (e.g. (ATKINS, 2016; Stanek et al., 2018)). This time-gap is achieved by calculating

9 the acceleration or deceleration of each of the dll controlled vehicles, for each simulation time

10 step, as follows: the acceleration or deceleration $(a)$ of a vehicle in VISSIM during each time

11 step is defined in equation (1): 
Papadoulis, Quddus, Imprialou

$a=\frac{\Delta v}{\Delta t}$

where $\Delta v$ is the difference between current speed and target speed and $\Delta t$ is the time step of the simulation, in this case $0.1 \mathrm{sec}$. Assuming that the dll controlled vehicle is not following the preceding vehicle with the desired time gap (d) - a situation which is graphically described in Figure 4- the distance travelled by both cars $\left(\mathrm{x}_{1}, \mathrm{x}_{2}\right)$ and the time gap during time step $\mathrm{t}$ and time step $t+0.1$ can be defined by equations (2)( 3 ) and (4)

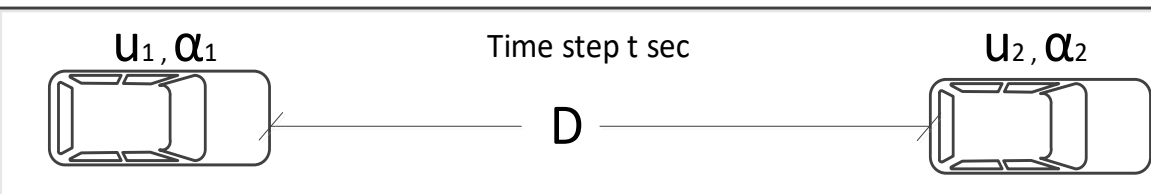

8

11

12

$$
x_{1}=u_{1} * t+\frac{1}{2} * a_{1} * t^{2}
$$

$$
x_{2}=u_{2} * t+\frac{1}{2} * a_{2} * t^{2}
$$

$$
d=x_{2}+D-x_{1}
$$

13 By subtracting equation (2) from equation (3) and taking into account that the target final 14 speed of the dll-controlled vehicle is the speed of the preceding vehicle, equation ( 5) and 15 subsequently ( 6) are calculated, assuming that the initial speed of the preceding vehicle $u_{2}$, 16 and the speed of the vehicle at the back, $u_{1}$ are not equal. In equation (6), $a_{1}^{\prime}$ represents the 17 acceleration of the CAV in order to achieve the desired time gap.

$$
t=\frac{2 *\left(x_{2}-x_{1}\right)}{u_{2}-u_{1}}
$$


Papadoulis, Quddus, Imprialou

$$
a_{1}^{\prime}=\frac{\left(u_{2}-u_{1}\right)^{2}}{2 *\left(x_{2}-x_{1}\right)}
$$

2 The dll-controlled vehicle continuously adjusts its acceleration according to equation (6). It

3 must be noted that the aforementioned acceleration/deceleration calculation only starts when the preceding vehicle in the same lane as the dll-controlled vehicle is a CAV vehicle.

\section{Lateral Movement}

This study hypothesises that when travelling on a motorway, CAVs will try to identify nearby CAVs in order to form a vehicle platoon (Haas and Friedrich, 2017; Gong and Du, 2018). If the preceding vehicle of the dll controlled vehicle is not a CAV, it searches adjacent lanes for nearby downstream CAV vehicles (see

Figure 3). The searching process was designed to resemble real-world sensor behaviour. In order to simulate sensor range in CAV vehicles, the radius that $\mathrm{CAV}$ vehicles could search for other CAV vehicles was set to 200 meters in the $\mathrm{C}++$ code, a value which was in-line with the range of a typical radar used in CAVs. During each time step, VISSIM creates a matrix containing the relative coordinates of the dll controlled vehicle with respect to nearby vehicles. The position of each nearby vehicle in the matrix depends on its relative simulation position to the dll-controlled vehicle and is described by two indexes: index 1, index 2 . Index 1 represents the lane that the nearby vehicle runs on; a value of zero means the nearby vehicle is in the same lane as the dll-controlled vehicle, a value of +1 and -1 means the nearby vehicle runs on the first lane on the left or right accordingly. Index 2 represents the relative longitudinal position compared to the dll-controlled vehicle; a value of +1 and -1 means the nearby vehicle is the next vehicle downstream and upstream accordingly whereas +2 or -2 means the nearby vehicle is the second next downstream or upstream accordingly. Consequently, that means that the maximum number of vehicles that can be tracked by the dll-controlled vehicle in a three-lane motorway is twelve and vehicles that are more than two vehicles away from the dll-controlled vehicle are not tracked.

Nearby vehicle data that could be made available to the dll-controlled vehicle included acceleration and deceleration, speed, distance, vehicle ID, lane angle, dimensions, and lane changing values, similar to the data gathered from a radar sensor. Adjacent CAVs could be identified from the dll-controlled vehicle due to a user-defined attribute programmed in the $\mathrm{C}++$ code.

If the first preceding vehicle in the same lane of the dll-controlled vehicle was not a CAV and another CAV was identified as a first preceding vehicle in an adjacent lane -starting from left to right-, a lane changing manoeuvre was initiated through the control algorithm, if the predefined time gap was found and the direction of the lane change was set. The required time gap that was set for the purpose of this study was 0.6 seconds from the vehicle upstream and downstream in the target lane. The lane changing parameters such as lane angle and number of target lane, are controlled entirely by VISSIM (PTV AG, 2010). Once the lane changing manoeuvre has been completed, the dll controlled vehicle engages CAV car-following behaviour. The step by step formulation of the vehicle platoons is schematically shown in

40 Figure 5 . Human driven vehicles are shown in green colour while the dll controlled vehicle 41 (CAVs) is shown in white. The dll controlled vehicle searches its preceding vehicles and identifies a CAV (shown in blue colour) in the lane on its left. Since a CAV is identified in an 
Papadoulis, Quddus, Imprialou

1 adjacent lane, the ego-vehicle then proceeds to search for a satisfactory gap for lane change.

2 After detecting the required gap, it initiates a lane change and joins the platoon.
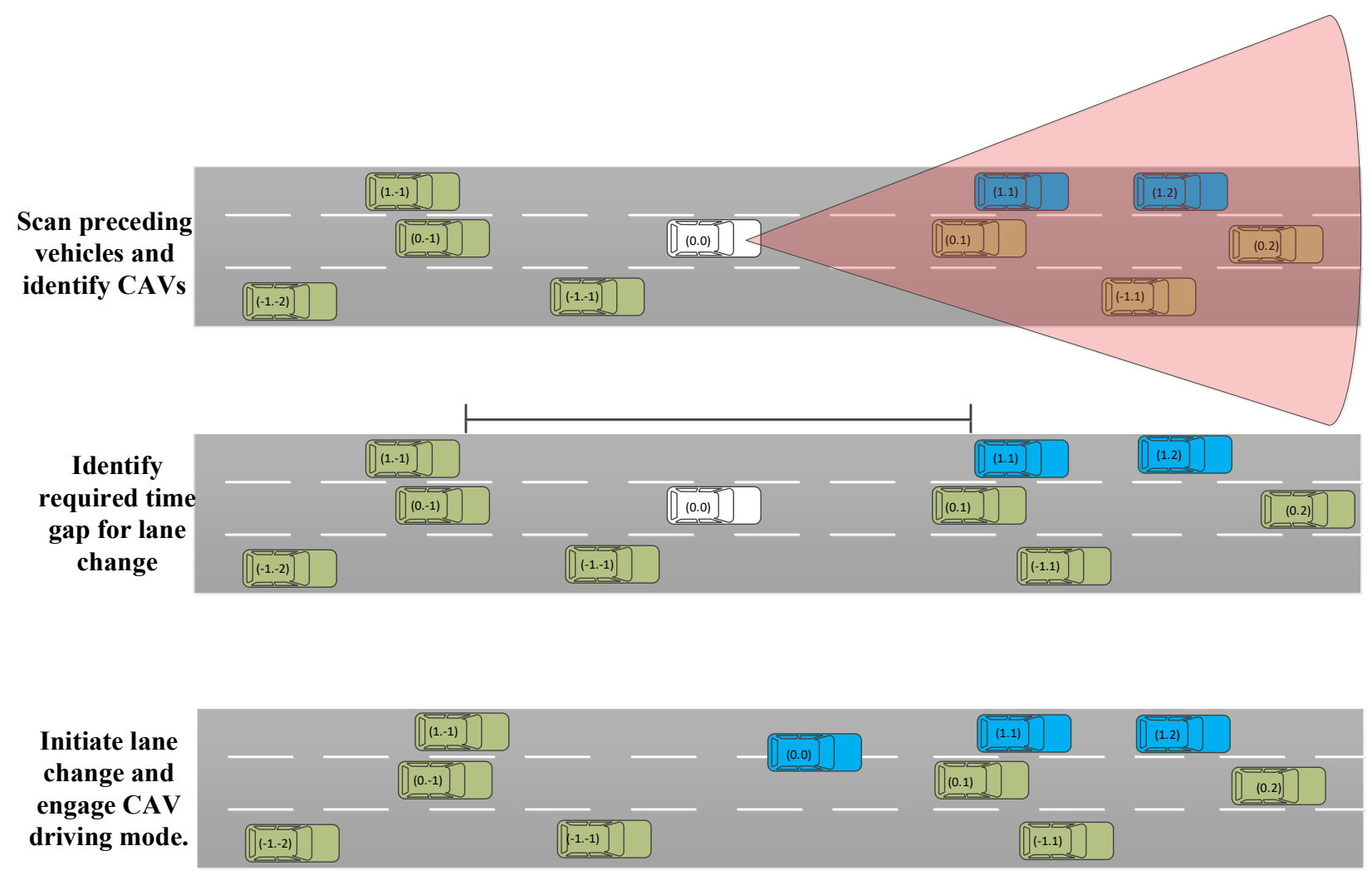

4 Figure 5 Step-by-step platoon formulation through the proposed CAV control algorithm

Vehicle Connectivity

Vehicle connectivity, in the form of network simulation (e.g. vehicular ad-hoc networks), cannot be directly achieved using only a traffic simulation package. However, according to the developed CAV behaviour, the range that CAVs could search in order to identify other CAVs, and obtain accurate acceleration/deceleration, speed measurements was programmed to be 200 meters.

It must be emphasized that this section presented an indirect simulation of the CAV subsystems (sensing, perception, planning and control) and behaviour, with reasonable assumptions. More specifically, the sensing subsystem was indirectly represented with the ability of the algorithm to collect nearby vehicles' data up to a range of 200 meters, while the perception subsystem was represented in the algorithm with the equations that transformed the raw data gathered, into useful variables that are used in the acceleration/deceleration calculation equations. The planning subsystem in this study was responsible for making the lateral-decision making decisions. Finally, the control subsystem was represented by the equations calculating the acceleration or deceleration of the CAV. 
Papadoulis, Quddus, Imprialou

\subsection{VISSIM and SSAM integration}

In order to evaluate the safety impact of CAVs, the Surrogate Safety Assessment Model (SSAM) is used in this study. SSAM is a tool developed and validated by the Federal Highway Administration of the U.S. Department of Transportation that utilises several algorithms to identify traffic conflicts from vehicle trajectory files (Gettman et al., 2008). VISSIM can output the vehicle trajectory file at the end of each simulation run by clicking the corresponding option in the VISSIM graphical user interface. The trajectory file includes data about the trajectory of all vehicles that appeared in the simulation. SSAM processes one simulation time step at a time and checks for traffic conflicts using predefined Time to Collision (TTC) and Post Encroachment Time (PET) threshold values. The default value for TTC is 1.5 seconds and for PET 5 seconds. While processing the vehicle trajectory files, SSAM projects the vehicles' future positions (next few simulation timesteps) if they are to keep the same speed and remain on the same path, for up to the duration of the predefined TTC value. If a vehicle overlap is identified in this way, this pair of vehicles is recorded in the SSAM output file. For more information on the identification of conflicts in SSAM, readers can refer to Gettman et al., (2008).

Along with the identification of the conflicting vehicles, SSAM provides data about the conflict itself such as the conflict type (i.e. rear-end, lane change or crossing), the simulation time when the minimum TTC value is observed, the coordinates of the location of the conflict, conflict angle and speed and acceleration values of the conflicting vehicles before and after the conflict. Finally, the latest version of SSAM (3.0) can provide heatmaps showing the concentration of conflicts along the simulated motorway segment.

\subsection{Data Collection, Calibration and Validation of the Microsimulation Model} Data Collection

The data used for calibration and validation come from two sources. The first one is historical, minute level, inductive loop detector traffic measurements between January 2016 and January 2018. The dataset comprised of 21,900 observations per inductive loop detector resulting in a total of 127 million observations. The data was split into a calibration (January 20162015 December 2016) and a validation dataset (January 2017-January 2018). Additionally, using this data, the vehicle fleet composition was calculated and used as input for VISSIM. More specifically, the heavy goods vehicles percentage from Monday to Friday was $20.02 \%, 21,47 \%$, $23,04 \%, 22,26 \%$ and $20.92 \%$ accordingly. The second source of data is traffic measurements collected by a Loughborough University's instrumented vehicle. The vehicle is equipped with a Continental ARS308-2 long range radar, a PointGrey Grasshopper 3 (GS3-U3-41C6C-C) camera, a Ublox NEO-M8L GPS, a Mobileye device and data are communicated through different devices using a CANbus. Real world trips between Junctions 19 and 21 were conducted between April 2017 and December 2017 with a total duration of 156 minutes with 140,400 observations. The data were divided equally into a calibration and a validation dataset. The data gathered from the trips were cleansed and fused together using Matlab.

\section{Calibration and Validation Strategy}

After taking into account previous safety simulation studies (Fan et al., 2013; Huang et al., 2013), a two-stage calibration approach was followed. The models developed, were calibrated for the times of the real-world trips; between 11:00 and 12:00 a.m. The number of simulations 
Papadoulis, Quddus, Imprialou

needed in order to achieve a 95\% confidence interval level for the simulation output was calculated using Equation (7) (Shahdah, Saccomanno and Persaud, 2015). In this equation, $N$ equals the required number of simulation runs, $\sigma$ equals the sample standard deviation of the simulation output, $t$ is the student's t-statistic for two-sided error of a $\alpha / 2$ with $N-1$ degrees of freedom and $E$ equals the allowed error range. The result showed that 15 simulation runs were sufficient and were conducted for each calibration and validation stage.

$$
N=\left(\frac{{ }^{t}\left(1-\frac{a}{2}\right), N-1}{E}\right)^{2}
$$

\section{i) First-stage calibration of VISSIM simulation model}

Initially, historical inductive loop detector data from the calibration dataset (minute by minute traffic flow, speed and headway distribution) were used as input for the simulation models. Data coming from the real-world inductive loop detectors were input at the corresponding simulation vehicle input point. In order to evaluate the performance of the proposed CAV algorithm under different traffic conditions, five simulation models were created, one for each weekday; Monday, Tuesday, Wednesday, Thursday and Friday. The average traffic flow values for the mainline corridor in each direction are presented in Figure 6.

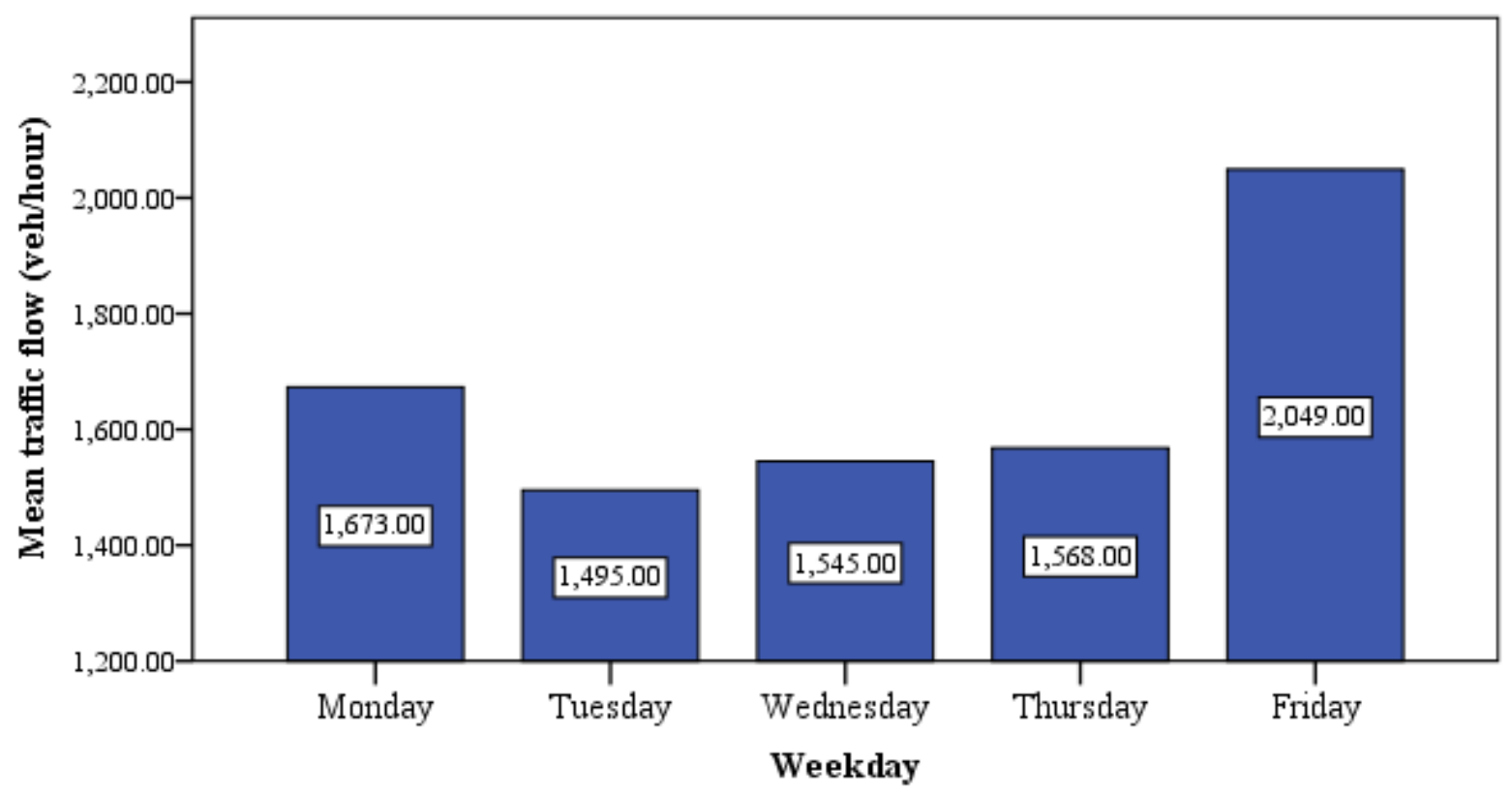

15

Figure 6 Average traffic flow per direction per weekday for the simulated time of the day

The first stage of the calibration was conducted to ensure that basic traffic parameters such as traffic volume, speed and travel time are accurately reproduced in the simulation. Following guidelines provided by FHWA (Dowling et al, 2004), the measures of performance chosen were travel time and traffic flow observed in the field versus the one produced in the simulation models. For travel time calibration, simulated values should be $\pm 15 \%$ of the observed values for more than $85 \%$ of the simulated cases. The travel time calibration results showed that the simulation model accurately represented the observed values and the results are presented in Figure 8. On the other hand, in order to calibrate traffic volume values, the GEH statistic is 
Papadoulis, Quddus, Imprialou

1 used. The $G E H$ statistic is presented in equation (8) where $E$ stands for the simulated traffic 2 volume and $V$ is the observed values. In order for the calibration to be successful, the $G E H$ 3 statistic should be less than 5 for $85 \%$ of the cases. The results of the calibration are presented 4 in Figure 8 and show that the simulation produces realistic traffic flow values. All the results 5 were validated using the validation dataset.

$$
G E H=\sqrt{\frac{(E-V)^{2}}{\frac{E+V}{2}}}
$$

6

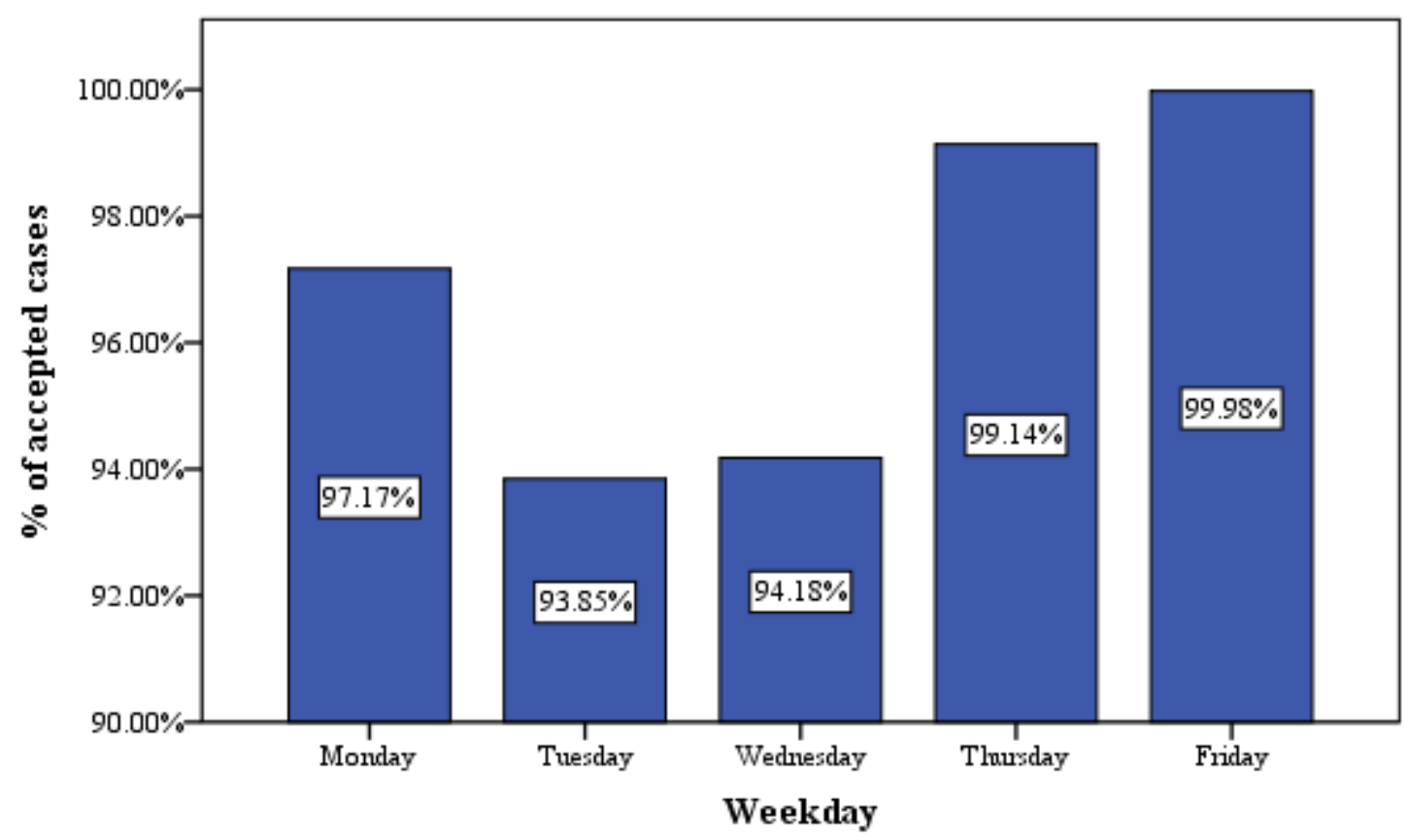

7

8 Figure 7 Travel time calibration results 
Papadoulis, Quddus, Imprialou

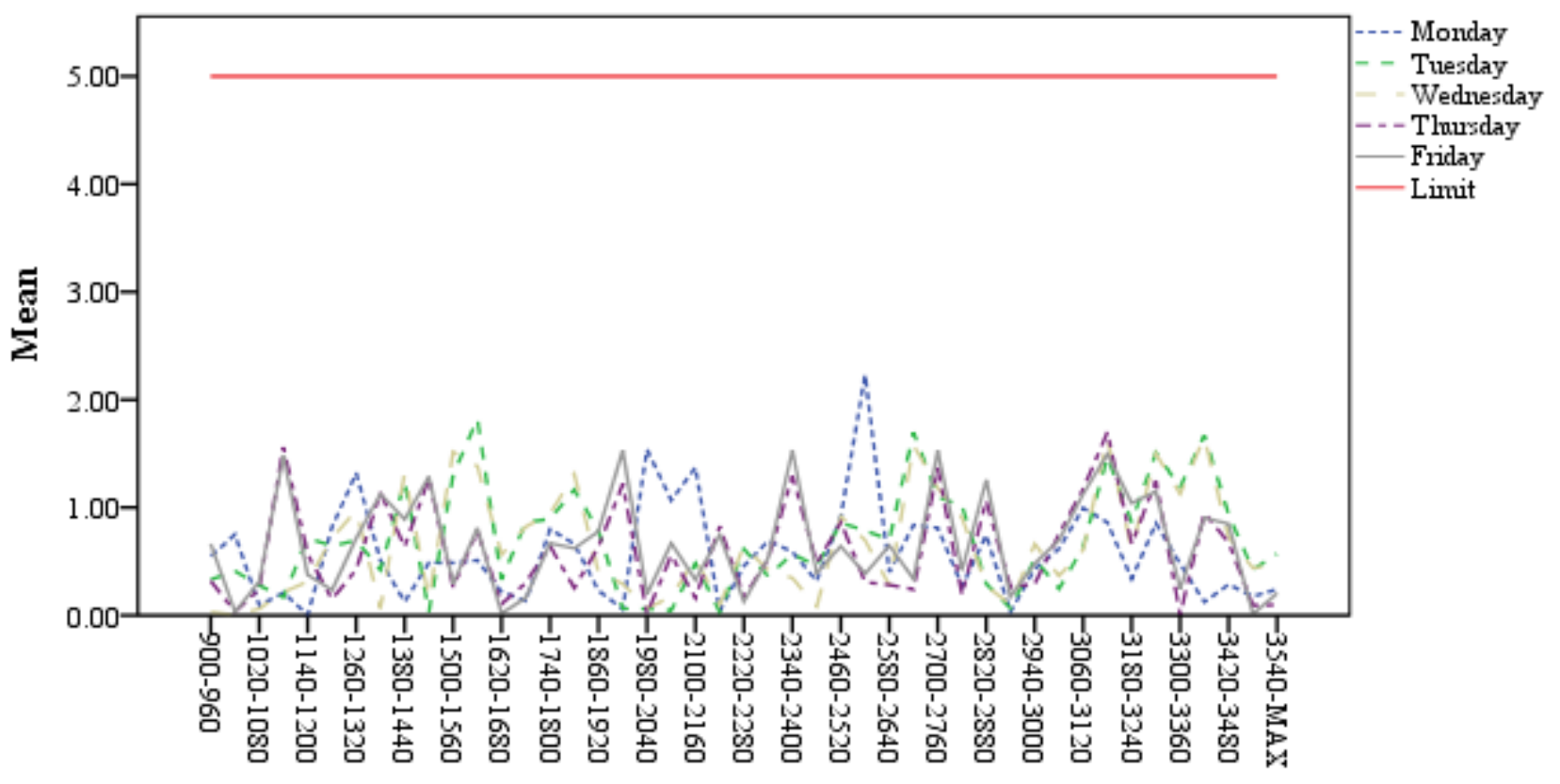

SimulationTime

Figure 8 Traffic volume calibration results

3

ii) Second-stage calibration of VISSIM simulation model

5

6

Subsequently, the second stage of the calibration ensured that the measure of safety was accurately simulated. The measure of safety chosen for this study was the Time To Collision (TTC) distribution. A TTC distribution was calculated from data gathered through Loughborough University's instrumented vehicle was compared with TTC distributions calculated from vehicles in VISSIM.

The instrumented vehicle data were used to extract TTC to the preceding vehicle in the same lane during the trips. The preceding vehicle was identified from the radar data since it had a small (less than $1.75 \mathrm{~m}$ ) lateral displacement to the ego-vehicle and had the smallest longitudinal displacement. Consequently, the distance and the relative velocity to the preceding vehicle were used in order to calculate the TTC surrogate safety measure, using its original definition which is presented in equation (9), where $\Delta X$ is the gap between the ego-vehicle and its preceding vehicle and $\Delta S$ is the difference of ego speed minus the speed of the preceding vehicle. The TTC distribution calculated from the calibration dataset set was used to calibrate the simulation model and the rest of the data was used for validation.

$$
T T C= \begin{cases}\frac{x_{l}-x_{f}-L_{l}}{v_{l}-v_{f}} & \text {, if } v_{F}>v_{L} \\ \infty & \text { if } v_{f \leq} v_{L}\end{cases}
$$

Since VISSIM does not provide TTC distributions by default, the TTC distribution for the simulation vehicles was calculated through an external code written in the External Driver Model API. The two distributions were compared using the non-parametric Mann-Whitney U 
Papadoulis, Quddus, Imprialou

test. Following guidelines from relevant literature (Habtemichael and Picado-Santos, 2013; Rahman and Abdel-Aty, 2018) and after testing all relevant car-following (CC0, CC2, CC3) and lane changing (Safety Distance Reduction Factor) parameter changes and several attempts, the change of the parameter CC3 (see Table 1) from -8 , which was the default value, to -5 provided the best $\mathrm{p}$-value for the Mann-Whitney test. With this change, the Mann-Whitney tests indicated that the two distributions were not significantly different (Significance values $0.875,0.716,0.611,0.127$ and 0.917 for Monday, Tuesday, Wednesday Thursday and Friday models accordingly). The results were validated using the validation data set.

It must be noted that the simulation model was calibrated and validated only for the humandriven vehicles ( $0 \%$ CAV market penetration rate), due to lack of CAV data. Once CAV data become available, a CAV calibration and validation could be achieved. However, calibrating the human driven model is critical, as it provides a solid foundation for the simulation models. Additionally, the human behaviour during the transition era will determine the reaction to CAVs and affect the safety impact of CAVs significantly.

\section{FINDINGS AND DISCUSSION}

The reduction of conflicts due to CAVs was evaluated using VISSIM and SSAM as described in the method section of the paper. In VISSIM, five different CAV market penetration scenarios were tested; $0 \%, 25 \%, 50 \%, 75 \%, 100 \%$. For each market penetration scenario and different simulation model (Monday-Friday), 15 simulation runs were performed with different random seeds. Each simulation run lasted 3,600 simulation seconds with 800 simulation seconds warm up period to allow the motorway segment to be fully occupied. It must be noted that as CAV market penetration rate increased, the computational time for each simulation run increased. For instance, a simulation run for the $0 \%$ market penetration rate scenario took approximately 30 minutes to finish while a simulation run for the $100 \%$ market penetration rate scenario took 45 minutes on average. The vehicle trajectory files produced by VISSIM were subsequently transferred to SSAM to identify traffic conflicts using TTC and Post Encroachment Time (PET) as surrogate safety measures.

SSAM identifies traffic conflicts using specific TTC and PET thresholds. The default threshold values are 1.5 seconds and 5 seconds respectively. The same values were used in this study following recommendations found in $\mathrm{Lu}$ et al, (2005) for medium and high collision risk situations. However, it is still unknown which TTC value would be critical for CAV driving. Only rear end and lane changing conflicts were taken into account according to the manual of SSAM for motorway scenarios (Pu and Joshi, 2008). It must be noted that even though the results for TTC threshold value of 1.5 seconds are presented below, a sensitivity analysis for TTC values of 1.0, 1.5, 2.0, 2.5 and 3 seconds was conducted and the percent reduction of conflicts per market penetration rate scenario was not affected significantly.

Firstly, the percent reduction of conflicts calculated by SSAM and the percent of conflicts related with CAVs for each of the pre-defined market penetration rates is presented in Table 2. The reduction of the total number of conflicts per weekday per scenario is graphically shown in Figure 9. A major reduction of conflicts is clear even at small market penetration rates. A small number of conflicts is observed at the $100 \%$ market penetration rate scenarios. These few conflicts could happen due to imperfections in the simulation software or be a consequence of a slow speed lane-changing manoeuvre. It is noticeable that the safety performance of the 
Papadoulis, Quddus, Imprialou

1 algorithm at the $25 \%$ market penetration rate is better as traffic flow increases. For example, 2 on Fridays, that the traffic flow is the highest, a greater reduction of conflicts is observed at the $325 \%$ market penetration rate than lower traffic weekdays. The reduction of conflicts at the $100 \%$ 4 market penetration rate varies between 90-94\%. At first glance, this reduction in simulated 5 conflicts seems to be very close with the anticipated safety benefit of CAVs according to the 6 literature (Fagnant and Kockelman, 2015; Kim et al., 2015). However, the results should not 7 be considered identical. A reduction of $90-94 \%$ in traffic conflicts which was calculated in this 8 study does not necessarily imply a 94\% reduction in crashes which was predicted in the

9 literature. An attempt to model the relationship between conflicts and crashes can be found in 10 Gettman et al., (2008). Furthermore, a possible explanation for the relatively small 11 improvement from $75 \%$ to $100 \%$ market penetration rates could be the fact that even from $75 \%$, 12 CAVs tended to form long platoons (8+ vehicles), isolating human driven vehicles in their own 13 lanes and making the interactions between human driven vehicles minimal. Finally, it is 14 observed that percentage of conflicts involving CAVs is significantly lower than the 15 corresponding market penetration rate.

16 Table 2 SSAM conflicts per CAV market penetration scenario

\begin{tabular}{ccccccc}
\hline \multicolumn{5}{c}{ Total Conflict Reduction \% } & & $\begin{array}{c}\text { \% conflicts } \\
\text { involving } \\
\text { CAVs }\end{array}$ \\
\hline $\mathbf{0 \%}$ & Monday & Tuesday & Wednesday & Thursday & Friday & - \\
\hline $\mathbf{2 5 \%}$ & $42.71 \%$ & $15.09 \%$ & $12.19 \%$ & $43.30 \%$ & $46.81 \%$ & $4.85 \%$ \\
\hline $\mathbf{5 0 \%}$ & $72.06 \%$ & $54.56 \%$ & $50.96 \%$ & $72.53 \%$ & $80.34 \%$ & $14 \%$ \\
\hline $\mathbf{7 5 \%}$ & $91.56 \%$ & $82.92 \%$ & $82.19 \%$ & $91.21 \%$ & $91.04 \%$ & $40 \%$ \\
\hline $\mathbf{1 0 0} \%$ & $-94.29 \%$ & $-90.05 \%$ & $-91.78 \%$ & $-92.86 \%$ & $-94.32 \%$ & - \\
\hline
\end{tabular}


Papadoulis, Quddus, Imprialou

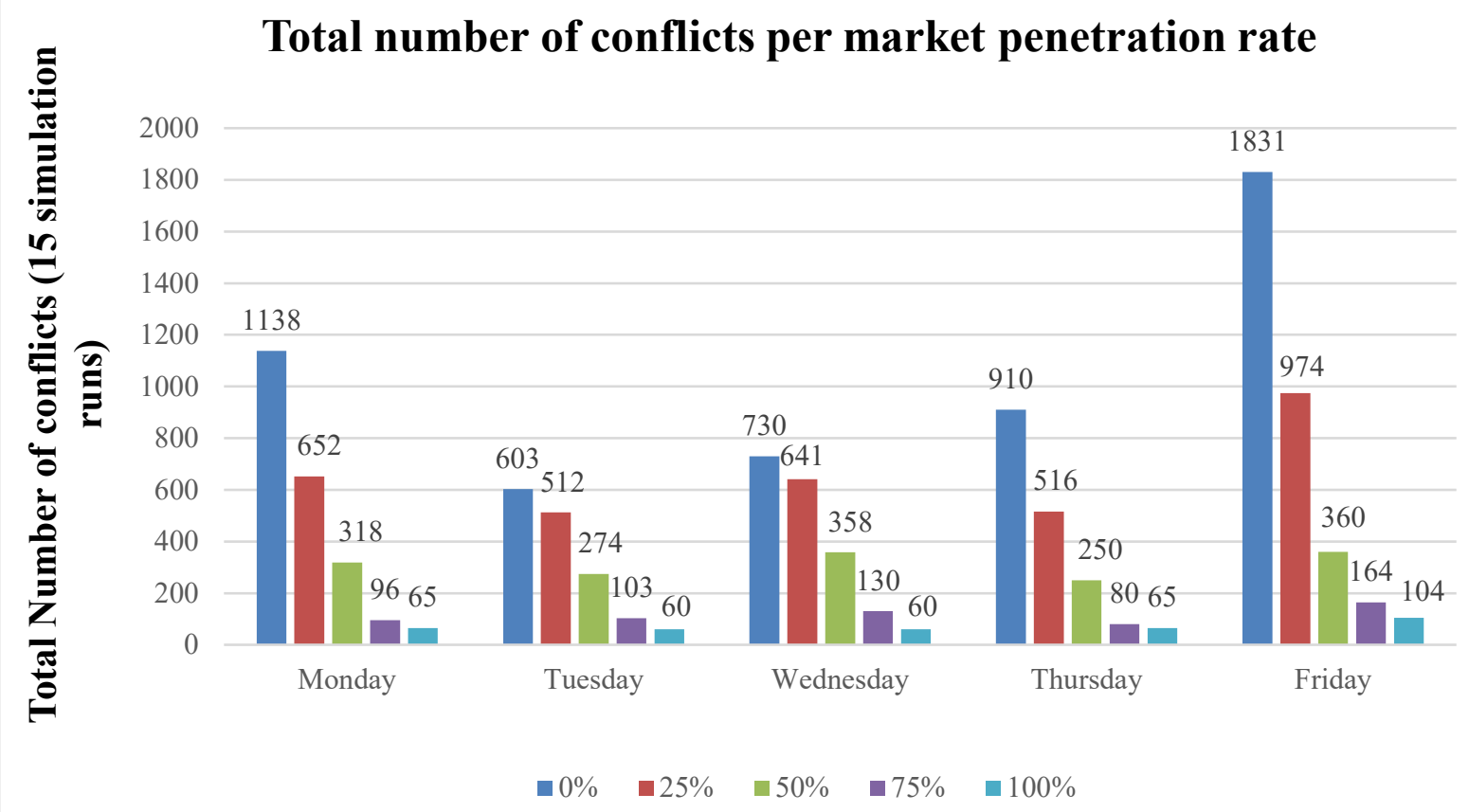

Figure 9 Reduction in traffic conflicts per market penetration scenario 3

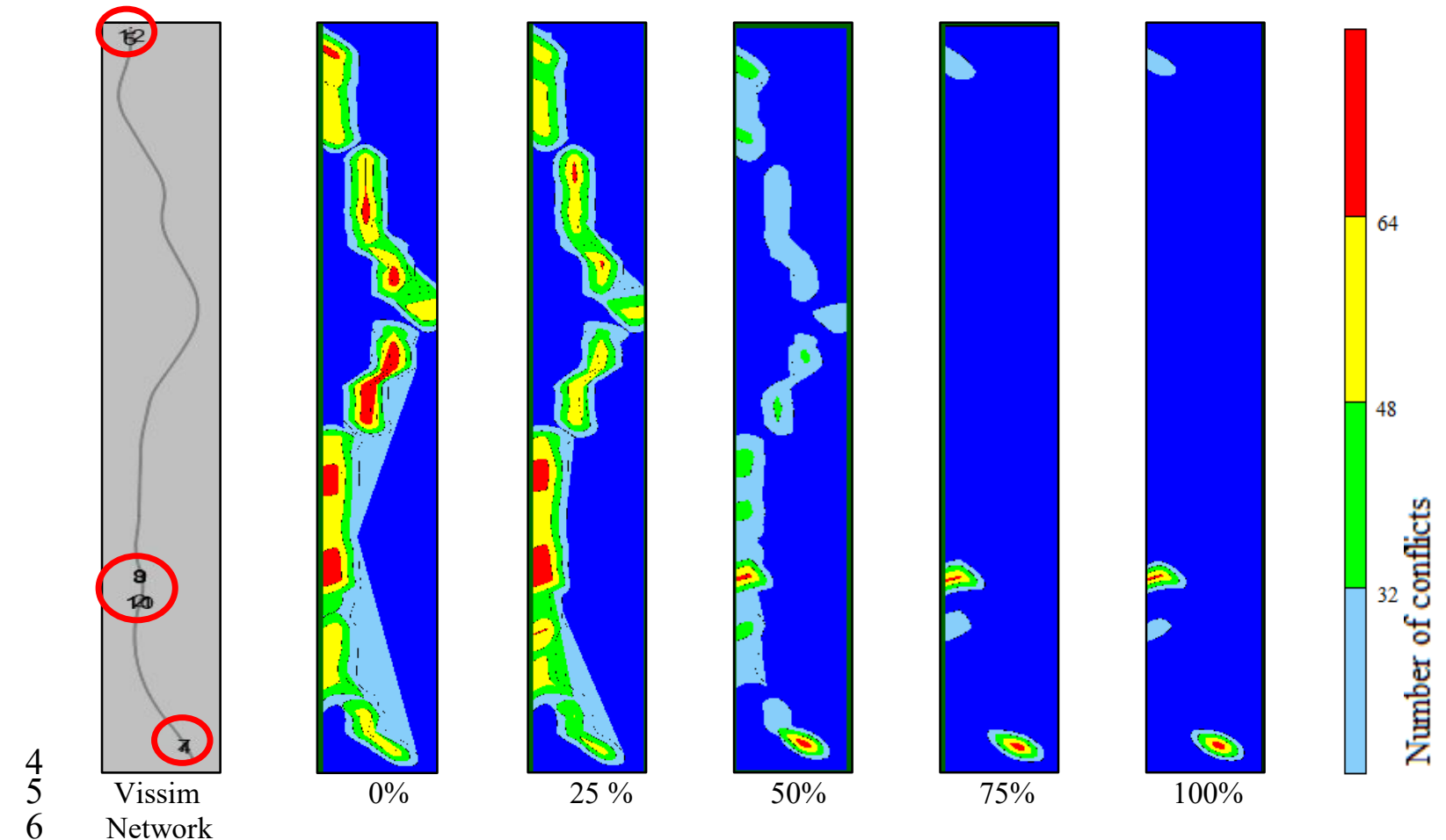

7 Figure 10 Heatmap of traffic conflict locations for the different market penetration scenarios 8 aggregated for all weekdays

10 Figure 10 presents the heatmaps showing the concentration of conflicts across the motorway 11 segment per market penetration scenario. It is obvious that the CAV control algorithm has 
Papadoulis, Quddus, Imprialou

eliminated conflicts in the non-merging/diverging areas in high market penetration rate scenarios. Inevitably, a high number of conflicts is observed at the merging and diverging areas (Junctions 21, 20 and 19 of the motorway, marked with a red circle in the left-most graph of Figure 10) due to the high variance of speeds and number of lane changes that take place on those segments. This finding seems to agree with relevant literature characterising the merging and diverging areas of the motorway as high risk segments (Ahammedet al 2008). Table 3 can provide more additional information about this issue; it provides the percentage of each conflict type per market penetration rate. The percentage of lane changing conflicts is coming close to the percentage of rear end conflicts as market penetration rate increases (although the absolute number of conflicts is reduced significantly). All the conflicts in the high market penetration rate scenarios (i.e. $75 \%$ and $100 \%$ ) are concentrated in or near the merging and diverging areas where lane changing behaviours are enforced. (see Figure 10) and that the lane changing conflicts happening in this area are a product of the actual lane changes and the rear-end conflicts are potentially a consequence of a lane change manoeuvre in this area.

\begin{tabular}{ccc}
\hline $\begin{array}{c}\text { Market } \\
\text { Penetration } \\
\text { Rate }\end{array}$ & Rear end & $\begin{array}{c}\text { Lane } \\
\text { change }\end{array}$ \\
\hline $0 \%$ & $9.18 \%$ & $90.82 \%$ \\
\hline $25 \%$ & $22.55 \%$ & $77.45 \%$ \\
\hline $50 \%$ & $23.26 \%$ & $76.74 \%$ \\
\hline $75 \%$ & $32.56 \%$ & $67.44 \%$ \\
\hline $100 \%$ & $43.68 \%$ & $56.32 \%$ \\
\hline
\end{tabular}

Table 3 Percentage of type of conflicts per market penetration rate scenario

The SSAM output indicated that in all market penetration rates, the minimum TTC value observed was 0 seconds, which implied a simulation crash. In some of the previous papers filter out these values claiming that they are caused by simulation errors (Gettman et al., 2008). However, others keep these virtual crashes in the analysis (Shahdah, Saccomanno and Persaud, 2015). After close observation of the behaviour of the vehicles in VISSIM some of these conflicts might have been caused due to simulation error; in the mainline vehicle input points, vehicles started a lane change at the same moment when another vehicle just entered the motorway resulting in a virtual crash. This problem was resolved by not allowing a lane change in the first 50 meters of the simulation network input points.

Finally, although the focus of this paper is to evaluate the safety impact of CAVs, in order to obtain more complete understanding of the impacts of CAVs, the travel time impact of the proposed algorithm was calculated as well and is presented in Table 4. CAVs seem to increase the average travel time during all weekdays due to the fact that long vehicle platoons with a slow leader decreased the average speed of the motorway significantly. This result however is sensitive to the desired speed distribution of vehicles in VISSIM. Overall, it is observed that, at the $100 \%$ market penetration scenario, CAVs managed to make the travel time almost equal across all weekdays. This means that CAVs will be able to provide reliable travel times independent of traffic conditions, a result that seems to agree with previous studies (ATKINS, 2016).

Table 4 Average travel time results for all vehicles for each CAV market penetration scenario 
Papadoulis, Quddus, Imprialou

\begin{tabular}{cccccc}
\hline & $\begin{array}{c}\text { Travel Time } \\
(\mathrm{sec})\end{array}$ & $\begin{array}{c}\text { Travel Time } \\
(\mathrm{sec})\end{array}$ & $\begin{array}{c}\text { Travel Time } \\
(\mathrm{sec})\end{array}$ & $\begin{array}{c}\text { Travel Time } \\
(\mathrm{sec})\end{array}$ & $\begin{array}{c}\text { Travel Time } \\
(\mathrm{sec})\end{array}$ \\
\hline $\mathbf{0 \%}$ & 727.28 & 751.0 & 748.3 & 796.9 & 822.2 \\
\hline $\mathbf{2 5 \%}$ & 820.88 & 805.1 & 804.0 & 830.0 & 849.76 \\
\hline $\mathbf{5 0 \%}$ & 843.01 & 835.1 & 835.3 & 848.8 & 858.30 \\
\hline $\mathbf{7 5 \%}$ & 861.89 & 859.3 & 859.4 & 864.2 & 866.00 \\
\hline $\mathbf{1 0 0} \%$ & 874.03 & 874.2 & 874.5 & 875.1 & 874.00 \\
\hline
\end{tabular}

With the results being presented, the limitations of this study should be taken into consideration. As real-world CAV data are not widely accessible, the behaviour of CAVs within a network is still unknown and validating simulated CAV behaviour is not possible. This study hypothesizes a CAV behaviour, that the CAV will be able to follow other CAVs more closely, search adjacent vehicles and try to form vehicle platoons. Furthermore, CAV safety in this study is assumed to be identical as human driving safety. Calculating conflicts with the same TTC threshold for CAVs might be not be a suitable safety surrogate as CAVs ideally should be more in control of their movement in relation to the surroundings, than conventional vehicles. The safety of CAVs in the road is strongly connected to software and hardware reliability (Pinto, 2012). This study does not consider autonomous driving mode breakdown, neither errors to the autonomous driving due to hardware malfunction or to physics-based causes.

As far as CAV traffic characteristics are concerned, this paper assumed that CAVs would want to mimic human throttle control and breaking behaviour, in order for the passengers to feel safe and comfortable. Hence, no special CAV desired acceleration-deceleration limits were used. However, the acceleration-deceleration values of CAVs were within the human driving values. Moreover, there was no limit to the length of the platoon that CAVs could form in the motorway in this study. This resulted in the formulation of long platoons (15+ vehicles), especially in high market penetration rates. A vehicle platoon this long could potentially cause several disturbances in the traffic flow of a motorway environment; e.g. blocking a vehicle from exiting the motorway at the merging/diverging areas or making it hard for human driven vehicles to navigate around them. Due to the non-congested traffic conditions tested in this paper, such problems were not observed in the simulation. Vehicles were able to change lane in advance in order to merge in and exit the motorway without causing traffic instability problems and platoons were able to dissolve at the off ramps without a problem. Additionally, the human driven vehicles seemed to adapt their driving behaviour due to vehicle platoons resulting in smaller speed variance in the motorway segment. Nonetheless, the authors are considering implementing a limit to the platoon length and possibly limiting the platoon formulation in certain lanes. However, it is still uncertain whether the platoon size will be limited in the real world when the CAV market penetration reaches high percentages and whether platoons will be allowed to form in specific lanes only. Finally, the authors are also looking for further improvements to the CAV control algorithm to optimize its performance and to resemble CAV behaviour more accurately.

The use of a validated CAV control algorithm could provide more trustworthy results, while more and better real world TTC data may improve the calibration of the model. A sensitivity analysis on the parameters of the CAV control algorithm such as time gap for both carfollowing, lane-changing situations and platoon size could be useful. Furthermore, adding a probabilistic term to data available to CAVs in VISSIM could represent a more dynamic CAV traffic environment. Finally, it would also be interesting to investigate the safety impact of CAVs in different scenarios namely a dedicated CAV lane in the simulation network or the re- 
Papadoulis, Quddus, Imprialou

routing of the CAV fleet in real time due to a disruption in the motorway. The limitations and concerns raised above, cover a narrow range of the spectrum of the challenges arising from the interaction between human driven vehicles and CAVs and mainly refer to a motorway environment. Further research is needed in order to fully investigate this interaction especially in more complicated (i.e.urban) road networks. Evaluating the safety impact of these competing traffic scenarios and traffic management would be the key in answering this important issue: where and when the safety benefit of CAVs is going to be maximised.

\section{CONCLUSION}

Simulating CAVs in order to evaluate their safety impact is a big challenge and this has not been widely addressed in existing literature. This paper developed a decision-making CAV control algorithm implemented in a simulated, real world motorway environment in the standard traffic simulation software VISSIM, using its External Driver Model Application Programming Interface. The algorithm included both longitudinal control and lateral decision making in which CAVs were able to search nearby vehicles in the motorway, identify nearby CAVs and by using the data available to them, change lanes in order to follow other CAVs with a shorter headway ( 0.6 seconds). Vehicles controlled by the API were able to calculate their acceleration at each simulation time step in order to keep a steady headway to other vehicles, ultimately forming vehicle platoons. A part of M1 motorway in the UK was simulated and five different simulation models were created, one for each day of the week (Monday to Friday) in order to test the CAV control algorithm in varying traffic conditions. The simulation model was calibrated and validated using real-world inductive loop detector data and time-tocollision (TTC) data calculated from radar measurements collected by the Loughborough University's instrumented vehicle. In order to investigate the effect of human driven vehicles - CAV interaction on safety as the technology is gradually adopted by road users, five different CAV market penetration rates were tested namely $0 \%$ (baseline), $25 \%, 50 \%, 75 \%$ and $100 \%$ and 15 simulation runs were conducted per scenario.

The vehicle trajectory files were consequently input to SSAM. SSAM calculated the total number of conflicts for each market penetration scenario and for each weekday using predefined TTC and PET thresholds. The results indicated that the CAV control algorithm improves road safety significantly, as the reduction of conflicts was $12-47 \%, 50-80 \%, 82-92 \%$ and $90-94 \%$ for the $25 \%, 50 \%, 75 \%$ and $100 \%$ penetration rates respectively. It was found that the algorithm was more effective at low market penetration rates on weekdays with higher traffic volume. Additionally, the reduction of conflicts achieved at $100 \%$ penetration rate seemed to agree with existing predictions in the literature. It was also apparent that CAVs provided reliable travel times regardless of traffic conditions. The results of this paper could provide useful initial insights to network operators about traffic conditions and road safety after the implementation of CAVs. The CAV control algorithm developed here could formally be integrated into a traffic microsimulation platform to simulate CAVs at a corridor-level in a mixed traffic stream and under different infrastructure and vehicle based scenarios.

\section{REFERENCES}

Ahammed, M. A., Hassan, Y. and Sayed, T. A. (2008) 'Modeling Driver Behavior and Safety on Freeway Merging Areas', Journal of Transportation Engineering, 134(9), pp. 370-377. doi: 10.1061/(ASCE)0733-947X(2008)134:9(370). 
Papadoulis, Quddus, Imprialou

1 Anderson, J. M. et al. (2014) 'The Promise and Perils of Autonomous Vehicle Technology', 2 in Autonomous Vehicle Technology: A Guide for Policymakers., pp. 12-16.

ATKINS (2016) Research on the impacts of connected and autonomous vehicles (CAVS) on traffic flow (results).

Dowling, R., Skabardonis, A. and Alexiadis, V. (2004) 'Traffic Analysis Toolbox Volume III : Guidelines for Applying Traffic Microsimulation Modeling Software', Rep. No. FHWAHRT-04-040, U.S. DOT, Federal Highway Administration, Washington, D.C, III(July), p. 146.

Essa, M. and Sayed, T. (2015) 'Transferability of calibrated microsimulation model parameters for safety assessment using simulated conflicts', Accident Analysis and Prevention. Elsevier Ltd, 84, pp. 41-53. doi: 10.1016/j.aap.2015.08.005.

Fagnant, D. J. and Kockelman, K. (2015) 'Preparing a nation for autonomous vehicles: Opportunities, barriers and policy recommendations', Transportation Research Part A: Policy and Practice. Elsevier Ltd, 77, pp. 167-181.

Fan, R. et al. (2013) 'Using VISSIM simulation model and Surrogate Safety Assessment Model for estimating field measured traffic conflicts at freeway merge areas', IET Intelligent Transport Systems, 7(1), pp. 68-77. doi: 10.1049/iet-its.2011.0232.

Figueiredo, M. C. et al. (2009) 'An Approach to Simulation of Autonomous Vehicles in Urban Traffic Scenarios', Proceedings of the 12th International IEEE Conference on Intelligent Transportation Systems, St. Louis, MO, USA, October 3-7, 2009, (June), pp. 322327. doi: 10.1109/ITSC.2009.5309524.

Gettman, D. et al. (2008) 'Surrogate Safety Assessment Model and Validation : Final Report', (June), p. FHWA-HRT-08-051.

Gong, S. and Du, L. (2018) 'Cooperative platoon control for a mixed traffic flow including human drive vehicles and connected and autonomous vehicles', Transportation Research Part B: Methodological. Elsevier Ltd, 116(October), pp. 25-61. doi: 10.1016/j.trb.2018.07.005.

Haas, I. and Friedrich, B. (2017) 'Developing a micro-simulation tool for autonomous connected vehicle platoons used in city logistics', Transportation Research Procedia. Elsevier B.V., 27, pp. 1203-1210. doi: 10.1016/j.trpro.2017.12.084.

Habtemichael, F. and Picado-Santos, L. (2013) 'Sensitivity analysis of VISSIM driver behavior parameters on safety of simulated vehicles and their interaction with operations of simulated traffic', 92nd Annual Meeting of the Transportation ..., (2013), pp. 1-17.

Hayes, B. (2011) 'Leave the driving to it', American Scientist, (99.5), pp. 362-366.

Huang, F. et al. (2013) 'Identifying if VISSIM simulation model and SSAM provide reasonable estimates for field measured traffic conflicts at signalized intersections', Accident Analysis and Prevention. Elsevier Ltd, 50, pp. 1014-1024. doi: 10.1016/j.aap.2012.08.018.

Jeong, E., Oh, C. and Lee, S. (2017) 'Is vehicle automation enough to prevent crashes? Role of traffic operations in automated driving environments for traffic safety', Accident Analysis \& Prevention. Elsevier, 104(February), pp. 115-124. doi: 10.1016/j.aap.2017.05.002. 
Papadoulis, Quddus, Imprialou

1 Kim, K. et al. (2015) An analysis of expected effects of the Autonomous Vehicles on

2 Transport and Land use in Korea Working Paper.

3 Li, Z. et al. (2013) 'Modeling Reservation-Based Autonomous Intersection Control in

4 VISSIM Modeling Reservation-Based Autonomous Intersection Control in VISSIM',

5 Transportation Research Record: Journal of the Transportation Research Board, 2381, pp.

6 81-90. doi: 10.3141/2381-10.

7 Lu, J. J., Pirinccioglu, F. and Pernia, J. C. (2005) 'Safety Evaluation of Right-Turns Followed

8 by U-Turns at Signalized Intersection (Six or More Lanes) as an Alternative to Direct Left

9 Turns: Conflict Data Analysis'. Available at:

10 http://www.fdot.gov/research/Completed_Proj/Summary_TE/FDOT_BC353_39_rpt_1.pdf.

11 Noort, M. Van, Arem, B. Van and Park, B. B. (2010) 'MOBYSIM: an integrated traffic 12 simulation platform', in Intelligent Transportation Systems (ITSC), 2010 13th International 13 IEEE Conference, pp. 1301-1306.

14 O'Hara, N. et al. (2012) 'MDDSVsim: an integrated traffic simulation platform for 15 autonomous vehicle research', in International workshop on Vehicular Traffic Management 16 for Smart Cities (VTM), p. 6.

17 Park, H. et al. (2012) 'Investigating Benefits of IntelliDrive in Freeway Operations : Lane 18 Changing Advisory Case Study', 138(September), pp. 1113-1122. doi:

19 10.1061/(ASCE)TE.1943-5436.0000407.

20 Pereira, J. L. F. and Rossetti, R. J. F. (2012) 'An integrated architecture for autonomous 21 vehicles simulation', Proceedings of the 27th Annual ACM Symposium on Applied 22 Computing-SAC '12, pp. 286-292.

23 Pinto, C. (2012) 'How Autonomous Vehicle Policy in California and Nevada Addresses 24 Technological and Non-Technological Liabilities', 5(1), pp. 1-16.

Poczter, S. L. and Jankovic, Lu. M. (2014) 'The Google Car : Driving Toward A Better

26 Future ?', Journal of Business Case Studies - First Quarter 2014, 10(1), pp. 7-14.

PTV AG (2010) Interface description DriverModel DLL, DriverModel DLL interface description.

PTV AG (2015) 'Ptv Vissim 8 User Manual'.

Pu, L. and Joshi, R. (2008) 'Surrogate Safety Assessment Model (SSAM): Software User Manual’, (May), p. 96. doi: FHWA-HRT-08-050.

Rahman, M. S. and Abdel-Aty, M. (2018) 'Longitudinal safety evaluation of connected vehicles' platooning on expressways', Accident Analysis and Prevention. Elsevier, 117(December 2017), pp. 381-391. doi: 10.1016/j.aap.2017.12.012.

Reich, S. (2013) Report on Automated and Autonomous Vehicles and Managed Lanes,

36 Center for Urban Transportation Research.

37 Roncoli, C., Papamichail, I. and Papageorgiou, M. (2015) 'Model predictive control for 38 motorway tra ffi c with mixed manual and VACS-equipped vehicles', Transportation 39 Research Procedia. Elsevier B.V., 10(July), pp. 452-461. doi: 10.1016/j.trpro.2015.09.095. 
Papadoulis, Quddus, Imprialou

1 Saunier, N., Sayed, T. and Lim, C. (2007) 'Probabilistic Collision Prediction for Vision-

2 Based Automated Road Safety Analysis pems aong whichtar in dtai by computing

3 cntinuoslymthe cllo', in Proceedings of the 2007 IEEE Intelligent Transportation Systems

4 Conference, pp. 872-878.

5 Shahdah, U., Saccomanno, F. and Persaud, B. (2015) 'Application of traffic microsimulation

6 for evaluating safety performance of urban signalized intersections', Transportation Research

7 Part C: Emerging Technologies. Elsevier Ltd, 60, pp. 96-104. doi:

$8 \quad$ 10.1016/j.trc.2015.06.010.

9 Silberg, G. et al. (2012) Self-driving cars: The next revolution." White paper, KPMG LLP \&

10 Center of Automotive Research.

11 Singh, S. (2015) 'Critical reasons for crashes investigated in the National Motor Vehicle

12 Crash Causation Survey. (Traffic Safety Facts Crash•Stats). Washington, DC: National

13 Highway Traffic Safety Administration.', in 13th International IEEE Conference on

14 Intelligent Transportation Systems, pp. 2-3.

15 Stanek, D. et al. (2018) 'Measuring Autonomous Vehicle Impacts on Congested Networks

16 Using Simulation', in Transportation Research Procedia.

17 Tarko, A. P. (2005) 'Estimating the frequency of crashes as extreme traffic events', Annual

18 Meeting of the Transportation Research Board, Washington, DC, (765), pp. 1-29.

19 Waymo (2016) Report on Autonomous Mode Disengagements for Waymo Self-driving

20 Vehicles in California.

21 Whaley, M. (2016) Developing Freeway Merging Calibration Techniques For Analysis of 22 Ramp Metering In Georgia Through VISSIM Simulation. 